\title{
Editorial: The second international workshop on health natural language processing (HealthNLP 2019)
}

\author{
Yanshan Wang ${ }^{1}$, Hua $\mathrm{Xu}^{2^{*}}$ and Ozlem Uzuner ${ }^{3}$ \\ From The second International Workshop on Health Natural Language Processing (HealthNLP 2019) \\ Xi'an, China. 10 June 2019
}

Keywords: Natural language processing, NLP, Healthcare, Electronic health records, EHR, Artificial intelligence

\section{Background}

In the past few decades, growing adoption of electronic health record (EHR) systems has made massive clinical narrative data available electronically. Natural language processing (NLP) technologies that can unlock information from narrative text have received great attention in the medical domain. Many clinical NLP methods and systems have been developed and showed promising results in various tasks. These methods and tools have also been successfully applied to facilitate clinical research, as well as to support healthcare applications. Recent advancements in artificial intelligence (AI), particularly deep learning-based neural networks, have achieved state-of-the-art performance on diverse NLP tasks in general domain, indicating great opportunities for solving real-world medical problems. At the same time, the amount of health information available online has exploded through use of social media, community forums, and health-related websites. These present additional challenges and opportunities for further development of new NLP methodologies and applications.

\section{HealthNLP workshop}

The goal of this workshop was to provide a unique platform to bring together researchers and practitioners working with health-related free text, and to facilitate close interaction among students, scholars, and industry professionals on health NLP challenges worldwide. We

\footnotetext{
* Correspondence: hua.xu@uth.tmc.edu

${ }^{2}$ School of Biomedical Informatics, The University of Texas Health Science

Center at Houston, Houston, TX, USA

Full list of author information is available at the end of the article
}

successfully organized the first international workshop on Health Natural Language Processing (HealthNLP 2018) in June, 2018, at New York City, USA [1].. We continued and held the HealthNLP 2019 workshop on June 10th, 2019, at Beijing, China, in conjunction with the IEEE International Conference on Healthcare Informatics (ICHI 2019). The workshop attracted submissions in the form of research papers, poster abstracts, and demonstration papers. All submissions were subjected to rigorous peerreview, with at least two peer-reviews and at least one review by a senior member of the program committee. Selected papers and abstracts were featured as oral / poster presentations at the workshop. We selected and invited eight high-quality submissions to extend their workshop abstracts for this journal supplement.

\section{Topics}

The main focus of the included papers is information extraction from clinical documents using deep learningbased approaches.

Heo et al. [2] proposed a hybrid ranking method that combines a co-occurrence approach considering both direct and indirect entity pair relationship with specialized word embeddings for measuring the relatedness of two entities. They evaluated the proposed ranking method with other well-known methods such as cooccurrence, Word2Vec, COALS, and random indexing by calculating top entities related to Alzheimer's disease. Furthermore, they conducted analysis of gene, pathway, and gene-phenotype relationships and found that the proposed method could find more hidden relationships than the traditional methods. 
$\mathrm{Li}$ and Hou [3] integrated the attention mechanism into a neural network, and proposed an improved clinical named entity recognition method for Chinese electronic medical records called BiLSTM-Att-CRF. Medical dictionaries and part-of-speech (POS) features were also introduced. They evaluated the proposed model on China Conference on Knowledge Graph and Semantic Computing (CCKS) 2017 and 2018 Chinese EMRs corpora, and found the model achieved better performance than other widely-used models. Their work preliminarily confirmed the validity of attention mechanism in extracting information from clinical documents.

In $\mathrm{Xu}$ et al.'s study [4], they adopted Bidirectional Long Short-Term Memory (BiLSTM) networks and Conditional Random Fields (CRF) to simultaneously identify named entity attributes, and to relate medical concepts to their attributes. Their approach achieved higher accuracy than the traditional systems that tackle two tasks separately on three medical concept-attribute detection tasks: disease-modifier, medication-signature, and lab test-value. They provide a simple yet unified solution to concept-attribute detection without using external data or knowledge bases, and thus streamlined practical clinical NLP systems.

De-identification of clinical notes is one of the most crucial prerequisites for utilizing clinical notes in other downstream biomedical informatics studies. Yang et al. [5] explored de-identification in cross-institute settings using deep learning-based approaches: fine-tuning and pre-training. They pre-trained de-identification models, LSTM-CRF, on the University of Florida (UF) Health corpus and fine-tuned the models on $\mathrm{i} 2 \mathrm{~b} 2$ datasets. They demonstrated that fine-tuning pre-trained models with a small local corpus (i.e., notes from UF Health) could significantly enhance the performance.

Wang et al. [6] developed and evaluated a rule-based NLP system to capture information on stage, histology, tumor grade and therapies in lung cancer patients using various clinical narrative documents including clinical notes, pathology reports and surgery reports. Their evaluation of the system showed promising results with precisions and recalls for stage, histology, grade, and therapies. They used convolutional neural networks $(\mathrm{CNN})$ in the error analysis, and found that CNN and the proposed NLP system could identify more true labels than the reference standard.

Li et al. [7] developed a disease classification algorithm for accurately recognizing rare diseases from symptom description documents. They leveraged a knowledge graph in representing documents and compared with LSTM models. On two Chinese disease classification data sets, the proposed algorithm delivered robust performance on rare diseases, outperforming a wide range of baselines, including resampling, deep learning, and feature selection methods.
A lack of publicly available clinical corpus resources has become a bottleneck for wide adoption of NLP applications in the clinical domain. Sun et al. [8] demonstrated a Chinese clinical corpus and a novel annotation work for chemical disease semantic extraction. The corpus is chronic disease specific and targeted at combination therapy related mining from biomedical abstracts in Chinese. The result analysis of the corpus verified its quality for the chemical-treat-disease relation identification task. The annotated corpus would be a useful resource for developing useful clinical relation extraction methods and tools.

\section{Discussion and conclusion}

In conclusion, the papers included in this special issue highlight the current research trends in health-related NLP field. With the successful applications of deep learning methods in the general domain, researchers have attempted to apply these methods to medical NLP tasks and have achieved promising results. We envision that these studies will have a significant impact on NLP methodologies, tools, and applications in the healthcare domain.

\section{Abbreviations \\ Al: artificial intelligence; BiLSTM: Bidirectional Long Short-Term Memory; CCKS: China Conference on Knowledge Graph and Semantic Computing; CNN: convolutional neural networks; CRF: Conditional Random Fields; EHR: electronic health record; HealthNLP: the workshop on Health Natural Language Processing; ICHI: the IEEE International Conference on Healthcare Informatics; NLP: natural language processing; POS: part-of-speech}

\section{Acknowledgements}

Not applicable.

\section{About this supplement}

This article has been published as part of BMC Medical Informatics and Decision Making Volume 19 Supplement 5, 2019: Selected articles from the second International Workshop on Health Natural Language Processing (HealthNLP 2019). The full contents of the supplement are available online at https://bmcmedinformdecismak.biomedcentral.com/articles/supplements/ volume-19-supplement-5.

\section{Authors' contributions}

YW, HX, and OU drafted the manuscript. All authors read and reviewed the final manuscript. All authors read and approved the final manuscript.

\section{Funding}

Not applicable.

Availability of data and materials Not applicable.

Ethics approval and consent to participate Not applicable.

Consent for publication

Not applicable.

Competing interests

The authors declare that they have no competing interests. 


\section{Author details}

'Department of Health Sciences Research, Mayo Clinic, Rochester, MN, USA. ${ }^{2}$ School of Biomedical Informatics, The University of Texas Health Science Center at Houston, Houston, TX, USA. ${ }^{3}$ Information Sciences and Technology, George Mason University, Fairfax, VA, USA.

\section{Published: 5 December 2019}

\section{References}

1. Vydiswaran VGV, Zhang Y, Wang Y, et al. Special issue of BMC medical informatics and decision making on health natural language processing. BMC Med Inform Decis Mak. 2019;19:76. https://doi.org/10.1186/s12911-0190777-0.

2. Go Eun Heo, Qing Xie and Min Song. A Hybrid Semantic Relatedness Algorithm by Entity Co-Occurrence and Specialized Word Embedding. BMC Med Inform Decis Mak. 2019;19(Supplement 5). https://doi.org/10.1186/ s12911-019-0934-5

3. Luqi Li and Li Hou. Combined Attention Mechanism for Named Entity Recognition in Chinese Electronic Medical Records. BMC Med Inform Decis Mak. 2019;19(Supplement 5). https://doi.org/10.1186/s12911-019-0933-6.

4. Jun Xu, Zhiheng Li, Qiang Wei, Yonghui Wu, Yang Xiang, Hee-Jin Lee, Yaoyun Zhang, Stephen Wu and Hua Xu. Applying a Deep Learning-Based Sequence Labeling Approach to Detect Attributes of Medical Concepts in Clinical Text. BMC Med Inform Decis Mak. 2019;19(Supplement 5). https:// doi.org/10.1186/s12911-019-0937-2.

5. Xi Yang, Tianchen Lyu, Chih-Yin Lee, Jiang Bian, William Hogan and Yonghui Wu. A Study of Deep Learning Methods for De-identification of Clinical Notes at Cross Institute Settings. BMC Med Inform Decis Mak. 2019;19(Supplement 5). https:/doi.org/10.1186/s12911-019-0935-4.

6. Liwei Wang, Lei Luo, Yanshan Wang, Jason A. Wampfler, Ping Yang and Hongfang Liu. Information Extraction for Populating Lung Cancer Clinical Research Data. BMC Med Inform Decis Mak. 2019;19(Supplement 5). https:// doi.org/10.1186/s12911-019-0931-8.

7. Xuedong Li, Yue Wang, Dongwu Wang, Walter Yuan, Dezhong Peng and Qiaozhu Mei. Improving Rare Disease Classification Using Imperfect Knowledge Graph. BMC Med Inform Decis Mak. 2019;19(Supplement 5). https://doi.org/10.1186/s12911-019-0938-1.

8. Yueping Sun, Li Hou, Lu Qin, Jiao Li and Qing Qian. RCorp:an Resource for Chemical Disease Semantic Extration in Chinese. BMC Med Inform Decis Mak. 2019;19(Supplement 5). https://doi.org/10.1186/s12911-019-0936-3.

\section{Publisher's Note}

Springer Nature remains neutral with regard to jurisdictional claims in published maps and institutional affiliations.

Ready to submit your research? Choose BMC and benefit from:

- fast, convenient online submission

- thorough peer review by experienced researchers in your field

- rapid publication on acceptance

- support for research data, including large and complex data types

- gold Open Access which fosters wider collaboration and increased citations

- maximum visibility for your research: over $100 \mathrm{M}$ website views per year

At $\mathrm{BMC}$, research is always in progress.

Learn more biomedcentral.com/submissions 